\title{
CARACTERIZAÇÃO DO DESCONFORTO FÍSICO RELACIONADO À ERGONOMIA EM PROFISSIONAIS DE ENFERMAGEM DO CENTRO CIRÚRGICO
}

\author{
CHARACTERIZATION OF PHYSICAL DISCOMFORT RELATED TO \\ ERGONOMICS IN NURSING PROFESSIONALS OF SURGERY CENTERS
}

\author{
Claudia Freire Brito', Livia Mara Gomes Pinheiro Correio²
}

Autora para correspondência: Claudia Freire Brito - cfreirebrito@hotmail.com 'Enfermeira pela Faculdade Independente do Nordeste (FAINOR). Vitória da Conquista, Bahia, Brasil. ${ }^{2}$ Enfermeira. Mestre em Ética e Gestão. Professora na Faculdade Independente do Nordeste (FAINOR). Vitória da Conquista, Bahia, Brasil.

\begin{abstract}
RESUMO | Objetivos: Caracterizar os profissionais de enfermagem que atuam no centro cirúrgico, identificar as queixas relacionadas ao desconforto físico decorrentes de riscos ergonômicos existentes no centro cirúrgico, bem como a sua localização anatômica, caracterizar e correlacionar as queixas com o ambiente de trabalho, além de verificar o tratamento e detectar as possíveis intervenções realizadas para prevenção dos riscos ergonômicos. Metodologia: Trata-se de um estudo exploratório de natureza quanti-qualitativa. A amostra foi constituída por 22 profissionais de enfermagem que atuam em centro cirúrgico. Resultados e Discussão: A maior parte dos participantes $(68,18 \%)$ respondeu que sente desconforto nos membros superiores, coluna ou nos membros inferiores, sendo que os locais mais citados como doloridos foram os ombros $(18,91 \%)$, coluna $(18,91 \%)$ e pernas $(18,91 \%)$. Os sintomas como a dor $(40,74 \%)$, dolorimento $(22,22 \%)$ e cansaço $(18,51 \%)$ foram as queixas mais registradas. Além disso, a maior parte dos participantes $(86,36 \%)$ informou que a unidade em que atuam não promove alguma intervenção para prevenção ou tratamento dos riscos ergonômicos e mais da metade dos participantes $(63,63 \%)$ informou que os problemas de desconforto que sentem estão relacionados ao trabalho no setor atual. Os profissionais sugeriram o melhor dimensionamento de pessoal e a fisioterapia preventiva como medidas importantes para prevenção de desconforto físico decorrente de problemas ergonômicos. Considerações finais: Vê-se necessidade da criação de um programa de prevenção de riscos ergonômicos no centro cirúrgico, como a estimulação da prática de atividade física, alongamentos, reeducação postural e outras atividades de fisioterapia preventiva.
\end{abstract}

\begin{abstract}
Objectives: characterizing nursing professionals who work in the surgery center, identifying the complaints related to physical discomfort arising from existing ergonomic risks in the surgery center, as well as its anatomical location, characterizing and correlating the complaints with the work environment, besides verifying the treatment and identifying possible interventions for preventing ergonomic risks. Methodology: This is an exploratory study with a quantitative and qualitative nature. The sample consisted of 22 nursing professionals who work in surgery centers. Results and Discussion: Most of the participants (68.18\%) answered that they feel discomfort in the upper limbs, spine or lower limbs, and the most cited places as painful were the shoulders $(18.91 \%)$, the column $(18,91 \%)$ and the legs $(18.91 \%)$. Symptoms such as pain $(40.74 \%)$, aching $(22.22 \%)$, and fatigue $(18.51 \%)$ were the most registered complaints. In addition, most of the participants $(86.36 \%)$ reported that the unit in which they work does not promote any intervention for prevention or treatment of ergonomic risks, and more than half of the participants $(63.63 \%)$ reported that the discomfort problems they feel are related to the work in the current sector. Professionals suggest a best personal design and a preventive therapy as important decisions for preventing the physical discomfort due to ergonomic problems. Final Thoughts: It is noticed the need of creating an ergonomic risk prevention program in the surgery centers, such as the stimulation of physical activity, the stretching, the postural re-education, and other preventive physiotherapy activities.
\end{abstract}

Keywords: Ergonomics, Discomfort, Nursing

Palavras-chave: Ergonomia, Desconforto, Enfermagem 


\section{INTRODUÇÃO}

A preocupação sobre a saúde dos trabalhadores de enfermagem cresceu bastante a partir dos anos 80 no Brasil. Existem fatores que determinam a vulnerabilidade e seu estado de saúde, estes dependem do local que o enfermeiro atua. Para caracterizar as peculiaridades do trabalho da enfermagem há de se analisar a composição da força de trabalho, formação técnica, formas de organização e divisão de trabalho, a predominância do sexo feminino a remuneração e etc ${ }^{1}$.

A ergonomia que é uma ciência aplicada que tem entre seus objetivos o de prevenir e diminuir acidentes e doenças do trabalho. De acordo com a International Ergonomics Association (IEA), é definida como: "O estudo da adaptação do trabalho ao homem ou, como a aplicação de conhecimentos científicos relativos ao homem e necessários para produzir ferramentas, máquinas e dispositivos que possam ser utilizados com o máximo de conforto, segurança e eficácia"2.

A saúde do trabalhador também depende das suas posturas adotadas durante o desempenho de suas atividades laborais. Qualquer tipo de estaticidade por um longo período de tempo, mesmo em um posto de trabalho confortável, é prejudicial. A posição básica que oferece risco ao trabalhador de enfermagem no centro cirúrgico é: posição em pé ou ortostática, na posição ereta o peso do corpo apoia-se na planta dos pés, para essa posição se tornar confortável é preciso inclinar um corpo um pouco para frente num ângulo de $10^{\circ}$ a $15^{\circ 3}$.

Cada componente da equipe de enfermagem possui atribuições no $\mathrm{CC}$, que podem ocasionar riscos ergonômicos a equipe ${ }^{4}$. A assistência de enfermagem prestada ao paciente, tanto no período que antecede a cirurgia quanto durante e após a realização da mesma, interfere nos resultados do procedimento realizado. Daí a relevância de buscar compreender a complexidade que envolve a atuação do enfermeiro nessa unidade. $O$ enfermeiro que atua em centro cirúrgico se relaciona com profissionais heterogêneos e este pode ser um dos fatores geradores de conflitos, divergências, insatisfações, evoluindo para o estresse ${ }^{5}$.
Devido a longas e continuas jornadas de trabalho pode-se causar diversas desordens relacionadas a desconforto físico, como problemas neuro-músculotendinosa, e nessa perspectiva apresentam-se as Lesões por Esforços Repetitivos (LER) ou Doenças Osteomusculares Relacionadas ao Trabalho (DORT) ou Afecções Musculoesqueléticas Relacionadas ao Trabalho (AMERT) que são definidas como doenças do trabalho que atinge tendões, sinoviais, músculos, nervos, faciais, ligamentos, de forma isolada ou associadamente com ou sem degeneração de tecidos, atingindo não somente os membros superiores, mas principalmente região escapular e pescoço ${ }^{3}$.

O trabalho no centro cirúrgico, trás consequências como absenteísmo, prejuízo para a qualidade das atividades de enfermagem, maior número de acidentes de trabalho, desinteresse no desenvolvimento profissional, apatia, refletindose diretamente na assistência de enfermagem Perioperatória. Diante disto, verifica-se a importância de haver uma mudança na forma de pensamento dos administradores ${ }^{6}$. Inapropriação de mobiliários e equipamentos; sustentação de posturas estáticas indevidas; trabalho com pacientes restritos ao leito podem infligir em desconfortos físicos que impactem em problemas de saúde nos profissionais. O diagnóstico precoce pode possibilitar orientações ergonômicas e posturais preventivas. O uso de aparelhos auxiliares, ajuste ocupacional, reorganização e ginástica laboral podem cooperar para "evitar a cronicidade e a incapacidade para o trabalhador"".

Diante do exposto, esta pesquisa teve como objetivo geral: identificar as queixas relacionadas a desconforto físico decorrentes de riscos ergonômicos existentes no centro cirúrgico, bem como a sua localização anatômica, caracterização e correlação com o ambiente de trabalho. E como objetivos específicos: caracterizar os profissionais de enfermagem que atuam no centro cirúrgico, além de verificar o tratamento e detectar as possíveis intervenções realizadas pela unidade para prevenção dos riscos ergonômicos. 
Trata-se de um estudo exploratório de natureza quanti-qualitativa. A coleta dos dados foi realizada em janeiro e fevereiro de 2016 no centro cirúrgico de um hospital privado de cunho filantrópico de grande porte de um município do interior da Bahia. Foram aplicados questionários semiestruturados com os participantes da pesquisa.

Para a seleção da amostra da pesquisa, foram utilizados como critérios de inclusão: profissionais de enfermagem - enfermeiros, técnicos e auxiliares de enfermagem (circulantes e instrumentadores) - que atuam no centro cirúrgico; com experiência profissional neste setor por no mínimo seis meses. $O$ número de participantes é referente ao quantitativo de profissionais que se disponibilizaram a participar da pesquisa, sendo que, do total de 28 que trabalham no setor, 4 estavam de férias durante o período de coleta de dados e 2 profissionais se recusaram a participar da pesquisa. Portanto, a amostra foi constituída por 22 profissionais.

Os dados, tanto qualitativos quanto quantitativos, foram obtidos com a aplicação do mesmo instrumento e depois foram sintetizados e transcritos para o Microsoft Word 2010. Os dados quantitativos foram dispostos em formato de tabelas após cálculo de porcentagem simples enquanto isso, os qualitativos referentes às sugestões dos participantes, foram submetidos à análise de conteúdo ${ }^{10}$.

O projeto foi aprovado pelo comitê de ética da Faculdade Independente do Nordeste - FAINOR, sob parecer $n^{\circ} 1.382 .990$. Para a concretização da coleta, todos os participantes da pesquisa assinaram o Termo de Consentimento Livre e Esclarecido (TCLE).

\section{RESULTADOS E DISCUSSÃO}

De acordo a tabela 1, a maioria dos participantes eram técnicas de enfermagem do sexo feminino $(77,27 \%)$, a faixa etária predominante foi entre 26 a 35 anos $(36,36 \%)$, e a metade dos participantes $(50,0 \%)$ trabalham em centro cirúrgico há no mínimo cinco anos.
Tabela 1. Caracterização dos participantes da pesquisa. Vitória da Conquista -BA, 2016.

\begin{tabular}{lcc}
\hline \multicolumn{1}{c}{ Variável } & $\mathbf{n}$ & $\%$ \\
\hline Sexo: & 5 & 22,73 \\
Masculino & 17 & 77,27 \\
Feminino & & \\
Profissão: & 2 & 9,09 \\
Enfermeiro & 18 & 81,81 \\
Técnico de enfermagem & 1 & 4,54 \\
Auxiliar de enfermagem & 1 & 4,51 \\
Instrumentador cirúrgico & & \\
Faixa Etária: & 5 & 22,72 \\
18 a 25 anos & 8 & 36,36 \\
26 a 35 anos & 7 & 31,81 \\
36 a 50 anos & 2 & 9,09 \\
Maior de 50 anos & & \\
Tempo de profissão: & 4 & 18,18 \\
O6 meses a 1 ano & 4 & 18,18 \\
1 a 3 anos & 3 & 13,63 \\
3 a 5 anos & 11 & 50,00 \\
5 anos ou mais & 22 & 100,00 \\
\hline TOTAL & & \\
\hline
\end{tabular}

Fonte: Dados da pesquisa (2016).

A predominância de mulheres na enfermagem possui causa histórica. $O$ cuidado desde o inicio era exercido por mulheres leigas que eram detentoras do cuidado doméstico às crianças, aos doentes e idosos, associado à figura de uma mulher-mãe que desde sempre foi curandeira e detentora de uma sabedoria informal de práticas de saúde. Assim a Enfermagem nasceu com características femininas e embora tenha se verificado um aumento significativo do número de homens nesta área, ainda é predominantemente feminina ${ }^{11}$.

Essa predominância do tempo de profissão prolongado (5 anos ou mais) justifica-se por este ser um setor especializado que geralmente exige tempo de experiência para atuação. Por isso, os profissionais são "peças" de difícil reposição, uma vez que é um setor com muitas especificidades para execução do trabalho' ${ }^{2}$.

De acordo resultados da tabela 2, a maior parte dos participantes $(68,18 \%)$ respondeu que sente desconforto nos membros superiores, coluna ou nos membros inferiores, sendo que os locais mais citados como doloridos foram os ombros $(18,91 \%)$, coluna $(18,91 \%)$ e pernas $(18,91 \%)$. É importante destacar que cada profissional pôde registrar mais de um local que sinta desconforto físico. 


\begin{tabular}{lcc}
\hline \multicolumn{1}{c}{ Variável } & n & \% \\
\hline $\begin{array}{l}\text { Você sente atualmente algum desconforto } \\
\text { físico nos membros superiores, coluna ou }\end{array}$ & & \\
membros inferiores. & & \\
Sim & 15 & 68,18 \\
Não & 7 & 31,82 \\
Se sim : & & \\
Pescoço(A) & 3 & 8,10 \\
Ombros (B) & 7 & 18,91 \\
Braços (C) & - & - \\
Coluna (D) & 7 & 18,91 \\
Cotovelos (E) & 1 & 2,70 \\
Antebraços (F) & 1 & 2,70 \\
Punho (G) & 1 & 2,70 \\
Mãos (H) & - & - \\
Quadril (I) & 2 & 5,40 \\
Coxas (J) & 1 & 2,70 \\
Joelhos (L) & 3 & 8,10 \\
Pernas (M) & 7 & 18,91 \\
Tornozelos/Pés (N) & 4 & 10,81 \\
\hline TOTAL & 37 & 100,0 \\
Fonte: Dados da pesquisa (2016) & &
\end{tabular}

Este grande número de queixas relacionadas a desconforto físico é preocupante, uma vez que demonstra risco potencial de problemas osteomusculares. Os locais de desconforto mais citados pelos participantes foram os mesmos encontrados em outro estudo ${ }^{13}$, também realizado com profissionais de centro cirúrgico.

Entre os locais mais predominantes, a dor na coluna foi destaque, e essa está comumente relacionada com causas mecânicos e posturais ${ }^{14}$. Portanto, as causas dessas dores e desconforto locais podem estar relacionadas com o posicionamento corporal inadequado do indivíduo durante a sua atividade laboral no centro cirúrgico.

Esses sintomas mais frequentes podem, a longo prazo, contribuir para $\circ$ surgimento de doenças relacionadas ao sistema musculoesquelético, que uma vez instaladas, estão entre os principais problemas de saúde pública e muitas vezes causam incapacidade para o trabalho, temporária ou permanente, bem como a geração de custos significativos para as organizações públicas, privadas e ○ Estado. As Lesões por esforço repetitivo e/ou Distúrbios Osteomuscular Relacionada ao Trabalho (LERDORT) se caracterizam pela ocorrência de vários sintomas, concomitantes ou não, tais como dor, parestesia, sensação de peso e fadiga de aparecimento insidioso, geralmente, na região cervical, cintura escapular e/ou membros superiores, mas podendo também acometer os membros inferiores ${ }^{15}$.

Dentre as profissões da área da saúde, a enfermagem, em particular, tem sido afetada pelos distúrbios musculoesqueléticos. Pesquisas realizadas em vários países mostram prevalências superiores a $80 \%$ de ocorrência destes distúrbios em trabalhadores de enfermagem. Já estudos brasileiros mostram prevalências de até $93 \%{ }^{15}$.

Conforme tabela 3, a maioria dos participantes $(63,63 \%)$ relacionaram os sintomas com o trabalho exercido no setor atual. Além disso, $45,45 \%$ das pessoas referiram que sentem desconfortos físicos há mais de seis meses. Outros sintomas como a dor $(40,74 \%)$, dolorimento $(22,22 \%)$ e cansaço $(18,51 \%)$ foram as queixas mais registradas. 
Tabela 3. Correlação do desconforto com o trabalho, tempo e caracterização dos desconfortos relatados pelos participantes da pesquisa. Vitória da Conquista - BA, 2016

\begin{tabular}{lcc}
\hline \multicolumn{1}{c}{ Variável } & $\mathbf{n}$ & $\%$ \\
\hline Os problemas de desconforto que você & & \\
se referiu, está relacionado ao & & \\
trabalho no setor atual. & 14 & 63,63 \\
Sim & 3 & 13,63 \\
Não & 5 & 22,72 \\
Não se aplica & & \\
Há quanto tempo. & - & - \\
Até 01 mês & 3 & 13,63 \\
De 01 A 03 meses & 3 & 13,63 \\
De 03 A 06 meses & 10 & 45,45 \\
Acima de 06 meses & 5 & 22,73 \\
Não se aplica & & \\
Qual é o desconforto. & 5 & 18,51 \\
Cansaço & 1 & 3,70 \\
Choques & - & - \\
Estalos & 6 & 22,22 \\
Dolorimento & 11 & 40,74 \\
Dor & 2 & 7,40 \\
Formigamento/Adoecimento & 1 & 3,70 \\
Peso & 1 & 3,70 \\
Perda de Força & - & - \\
Limitações de Movimentos & 27 & 100,0 \\
\hline TOTAL & & \\
\hline
\end{tabular}

Fonte: Dados da pesquisa (2016)

A correlação feita pela maioria dos profissionais quanto ao desconforto $e$ as atividades desempenhadas no centro cirúrgico, evidencia que esses têm consciência da existência de riscos para a saúde decorrente das suas atividades laborais. Outro dado que chama a atenção é que grande parte dos profissionais já convive com o desconforto físico durante longo período, o que é preocupante, uma vez que, em longo prazo, estes sintomas podem se agravar.

De acordo com estudo recente ${ }^{15}$, a dor é um importante sintoma osteomuscular que deve ser valorizado e investigado para que a terapêutica seja estabelecida e os fatores de risco sejam minimizados.

A maioria das queixas de saúde nos trabalhadores de enfermagem relaciona-se ao sistema osteomuscular, atribuídas principalmente a fatores ergonômicos e posturais inadequados, presentes na dinâmica hospitalar evidenciando a gravidade deste problema nesta categoria. Os sintomas físicos apresentados acima são frequentes na rotina de trabalho desses profissionais, principalmente devido à grande demanda de trabalho que comumente é exigida no centro cirúrgico: realização de procedimentos relativos à assistência de enfermagem, transporte e manipulação de pacientes, entre outras, assim como procedimentos relativos à gerência, como - preenchimento de impressos, as anotações em prontuário, realização de escalas e gráficos de movimento de pacientes ou de cirurgia, atividades que, de uma forma ou de outra, podem contribuir para a gênese de um distúrbio osteomuscular ${ }^{16}$.

No ambiente cirúrgico, existem vários fatores ergonômicos relacionados com problemas ambientais e organizacionais que podem ser relacionados às lesões osteomusculares, tais como recursos tecnológicos inadequados, incluindo mobiliário, a falta de equipamentos especiais para movimentar pacientes, além da escassez de recursos humanos e a falta de treinamento ${ }^{16}$.

Conforme resultados apresentados na tabela 4 , a maioria dos participantes $(68,18 \%)$ classificam a dor ou desconforto que sentiram como de intensidade moderada e $31,81 \%$ informaram aumento dos sintomas durante a jornada normal de 
trabalho. Além disso, as férias se destacaram como o período em que as pessoas sentem mais alívio dos sintomas $(36,26 \%)$; e a metade dos participantes $(50,0 \%)$ informou que fazem uso de algum tipo de remédios, emplastros ou compressas para aliviar os sintomas durante a jornada laboral.

Tabela 4. Intensidade, período e métodos para alívio a dor e outros desconfortos. Vitória da Conquista/BA, 2016.

\begin{tabular}{lcc}
\hline \multicolumn{1}{c}{ Variável } & n & $\%$ \\
\hline $\begin{array}{l}\text { O que sente você classifica como: } \\
\text { Muito Forte/Forte }\end{array}$ & 3 & 13,63 \\
$\begin{array}{l}\text { Moderado } \\
\text { Leve/Muito Leve }\end{array}$ & 15 & 68,18 \\
Não se aplica & - & - \\
O que você sente aumenta com o trabalho? & 4 & 18,18 \\
$\begin{array}{l}\text { Durante a Jornada de Trabalho } \\
\text { Durante as horas extras }\end{array}$ & 7 & 31,81 \\
Á noite & 4 & 18,18 \\
Não & 3 & 13,63 \\
Não se aplica & 4 & 18,18 \\
O que você sente melhora com o repouso? & 4 & 18,18 \\
A noite & & \\
Nos finais de semana & 4 & 18,18 \\
Durante o revezamento em outras tarefas & 2 & 9,09 \\
Férias & 2 & 9,09 \\
Não melhora & 8 & 36,26 \\
Não se aplica & 2 & 9,09 \\
Você tem tomado remédio ou colocado & 4 & 18,18 \\
emplastros/ compressas para poder & & \\
trabalhar? & & \\
Sim & 11 & 50,00 \\
Não & 7 & 31,31 \\
Não se aplica & 4 & 18,18 \\
\hline TOTAL & 22 & 100,0 \\
\hline
\end{tabular}

Fonte: Dados da pesquisa (2016)

Ficou evidente que a jornada de trabalho aumenta a intensidade da dor ou desconforto ${ }^{17}$. Isso, se deve ao fato de, no ambiente de trabalho, os profissionais continuam expostos aos riscos ergonômicos causadores dos problemas osteomusculares ${ }^{18}$.

A jornada de trabalho intensa a que muitos trabalhadores de enfermagem estão expostos contribui diretamente para 0 surgimento de desconforto físico, principalmente no contexto hospitalar, que é muito comum o sistema de plantões extensos, e de duplos cargos. Isso acontece devido à baixa remuneração a esses profissionais ${ }^{19}$.

A enfermagem é considerada uma profissão desgastante/estressante de acordo com estudos de vários autores ${ }^{20,21,22,23,24,25}$, que observam que estas situações sobrevém das condições extrínsecas ao enfermeiro, devido à atuação hospitalar com extensa carga de trabalho, responsabilidade por mais de um setor, proximidade a dor, sofrimento dos pacientes e de seus familiares, e intrínsecas à personalidade do enfermeiro e sua visão da morte. Estes também, orientam que as razões de desgaste devem ser verificadas e trabalhadas pela instituição e pelo enfermeiro, afim de se produzirem instrumentos em melhoria a saúde do trabalhador e do paciente por ele atendido.

Observa-se que os profissionais de enfermagem de centro cirúrgico têm suportado cargas de trabalho cada vez maiores, com proporção inadequada de pacientes por profissionais qualificados, turnos rotativos, baixa remuneração, manipulação de substâncias tóxicas e presença de fatores de risco pertinentes ao ambiente, levando a uma situação conhecida como sobrecarga de trabalho. Como conseqüência desta situação tem-se um alto grau de frustração e descontentamento em relação à responsabilidade e exercício profissional, podendo desencadear os transtornos físicos, psicológicos afetando sua saúde e levando a um comprometimento de sua qualidade de vida ${ }^{26}$. 
Conforme tabela 5, a maioria dos profissionais $(59,09 \%)$ informaram que nunca fizeram algum tratamento médico por algum distúrbio ou lesão em membros superiores, coluna ou membros inferiores. $E$ os que informaram tratamento, a hérnia de disco foi o distúrbio que mais se despontou como causa de tratamento médico $(55,55 \%)$. Além disso, a maior parte dos participantes $(86,36 \%)$ informou que a unidade em que atuam não promove alguma intervenção para prevenção ou tratamento dos riscos ergonômicos e mais da metade dos participantes $(63,63 \%)$ também informaram que o que sente seja dor, fadiga, desconforto muscular, insônia, etc está relacionado a atividades no trabalho e atrapalham sua qualidade de vida fora do trabalho.

Tabela 5. Tratamento e prevenção dos problemas decorrentes de riscos ergonômicos no centro cirúrgico. Vitória da Conquista/BA, 2016.

\begin{tabular}{|c|c|c|}
\hline Questão & $\mathbf{n}$ & $\%$ \\
\hline \multicolumn{3}{|l|}{$\begin{array}{l}\text { Você já fez tratamento médico alguma } \\
\text { vez por algum distúrbio ou lesão em } \\
\text { membros superiores, coluna ou } \\
\text { membros inferiores. }\end{array}$} \\
\hline $\operatorname{Sim}$ & 9 & 40,91 \\
\hline Não & 13 & 59,09 \\
\hline \multicolumn{3}{|l|}{ Para qual distúrbio. } \\
\hline Bucite & 2 & 22,22 \\
\hline Varizes & 1 & 11,11 \\
\hline Artrite & 1 & 11,11 \\
\hline Hérnia de Disco & 5 & 55,55 \\
\hline \multicolumn{3}{|l|}{$\begin{array}{l}\text { A unidade que você atua promove } \\
\text { alguma intervenção para prevenção ou } \\
\text { tratamento dos riscos ergonômicos. }\end{array}$} \\
\hline Sim & 3 & 13,64 \\
\hline Não & 19 & 86,36 \\
\hline $\begin{array}{l}\text { Na sua opinião, o que você sente seja } \\
\text { dor, fadiga, desconforto muscular, } \\
\text { insônia, etc relacionado a atividades no } \\
\text { trabalho, atrapalham sua qualidade de } \\
\text { vida fora do trabalho. }\end{array}$ & & \\
\hline $\operatorname{Sim}$ & 14 & 63,63 \\
\hline Não & 08 & 36,37 \\
\hline
\end{tabular}

Fonte: Dados da pesquisa (2016)

Entre os problemas de saúde decorrentes dos riscos ergonômicos, a hérnia de disco destacou-se como um grave problema de saúde para os trabalhadores, - que pode ter sido causado, conforme estudo de $2010^{14}$ devido às movimentações e as posturas incorretas decorrentes das inadequações do ambiente de trabalho, das condições de funcionamento dos equipamentos disponíveis, bem como das formas de organização e de execução do trabalho.

Quanto à qualidade de vida, conforme estudo com trabalhadores de enfermagem de centro cirúrgico ${ }^{26}$, os resultados apontam que a qualidade de vida se mostrou prejudicada nos domínios dor, vitalidade, aspectos sociais, aspecto físico e saúde mental, o que evidencia que a dor osteomuscular é um fator importante na qualidade de vida de trabalhadores de enfermagem cirúrgica.

Vê-se como informação preocupante a falta de medidas preventivas dos riscos ergonômicos no centro cirúrgico. Neste sentido, os gestores de saúde devem atentar-se para a necessidade da prevenção desses riscos, através de medidas de prevenção primária, como reeducação postural ocupacional. Isso é importante, uma vez que a adaptação ergonômica do ambiente de trabalho com eliminação de movimentos repetitivos é essencial, pois a limitação álgica impacta na qualidade do atendimento ${ }^{7}$.

Quando questionados sobre possíveis sugestões que poderiam ser implementadas para diminuir os riscos ergonômicos no centro cirúrgico, os profissionais apontaram diversas possibilidades que deveriam ser implantadas no setor, conforme falas abaixo: 
“... é preciso contratar mais funcionários e reduzir as horas de trabalho para diminuir a carga de trabalho P3

“... é urgente a necessidade de fazer inspeção das rodas das macas, pois, são antigas e devido ao atrito, aumenta o peso para a gente. Falta manutenção". P6

“... o temo de descanso é pouco. A gente não pára. É preciso programar isso melhor, para que a gente possa descansar um pouco. No fim do plantão, estou moída".

"acho que a fisioterapia laboral para os funcionários seria uma boa estratégia". P1

Conforme as sugestões dos profissionais, torna-se necessário avaliar o dimensionamento de pessoal de enfermagem para evitar cargas extensas de trabalho. Além disso, um programa de fisioterapia preventiva deve ser adotado para aperfeiçoar os resultados almejados. Alterações, como técnicas de elevação, manuseio e transporte de cargas, otimização da segurança no trajeto a ser percorrido com a mesma, devem ser adotadas. Os pés do profissional devem ser posicionados corretamente, sendo que a carga deve ser fixada com o apoio total das mãos. Ao levantar cargas do chão, com o dorso compensado e os joelhos fletidos, sendo a carga carregada o mais rente ao corpo. Movimentos de torção ao redor do eixo vertical corporal também devem ser evadidos ${ }^{7}$.

A Organização Mundial de Saúde acrescenta, ainda, os agentes ergonômicos decorrentes de má postura, inadequação do mobiliário e outros responsáveis por distúrbios osteomusculares; dos agentes psicossociais advindos de relações conflituosas, monotonia, ritmo excessivo, entre outros ${ }^{27}$.

Quanto ao mobiliário pode-se prevenir com bancadas e móveis variando, conforme o tamanho do trabalhador e o tipo de serviço executado. Devemse alternar os pés em um banquinho ao executar tarefas em ortostatismo prolongado. Alcances e 0 alongamento excessivo da coluna vertebral devem ser evitados. O conforto térmico, visual e acústico não pode ser desprezado, e um número mínimo de profissionais por plantão é imprescindível'.

Sempre que se mantém uma postura inadequada, sobrecarrega-se nossa coluna, fazendo com que os discos intervertebrais sofram cargas anormais. Desta forma, a postura correta consiste em uma forma de prevenção de doenças da coluna vertebral ${ }^{28}$.

O aumento do quantitativo de pessoal foi uma das soluções recomendadas pelos profissionais. Não raramente, a equipe de enfermagem que atua em instituições hospitalares que apresentam déficits de recursos humanos tornando a execução do trabalho mais penosa, gerando uma série de agravos a saúde ${ }^{16}$.

\section{CONSIDERAÇÕES FINAIS}

Diante dos resultados encontrados, viu-se que a maior parte dos profissionais que atuam em centro cirúrgico relatou sentir desconforto físico, especialmente dor de intensidade moderada, a qual é piorada durante a jornada de trabalho.

O estudo atendeu aos objetivos propostos pela pesquisa, uma vez que conseguiu caracterizar os profissionais de enfermagem que atuam no centro cirúrgico, com destaque para $\circ$ sexo feminino com tempo prolongado de profissão como predominante. Identificou também que a maioria dos participantes relatou sentir desconforto físico, com destaque para a região dos ombros, colunas e pernas. A dor, o dolorimento e o cansaço foram as queixas mais registradas e a maioria dos sintomas foram relacionadas com o trabalho atual.

Além disso, em consonância aos objetivos da pesquisa, ficou evidente que a maioria dos participantes não procura atendimento médico, $\circ$ que pode ocasionar o agravamento das queixas existentes. Outra informação preocupante foi que a unidade, segundo a maioria dos participantes, não promove alguma intervenção para prevenção ou tratamento dos riscos ergonômicos.

Perante esse problema, vê-se necessidade da criação de um programa de prevenção de riscos ergonômicos no centro cirúrgico, como a estimulação da prática de atividade física, alongamentos, reeducação postural e outras atividades de fisioterapia preventiva. Associado a isso, sugere- 
se a necessidade de adequação ergonômica do mobiliário e equipamentos do centro cirúrgico, de modo a minimizar os riscos ergonômicos geradores de desconfortos físicos que interfiram na qualidade de vida do profissional.

\section{CONFLITOS DE INTERESSES}

Nenhum conflito financeiro, legal ou político envolvendo terceiros (governo, empresas e fundações privadas, etc.) foi declarado para nenhum aspecto do trabalho submetido (incluindo mas não limitandose a subvenções e financiamentos, conselho consultivo, desenho de estudo, preparação de manuscrito, análise estatística, etc).

\section{REFERÊNCIAS}

1. Ribeiro EJG, Shimizu HE. Acidentes de trabalho com trabalhadores de enfermagem. Rev. bras. enferm. 2007;60(5):535-540. doi: 10.1590/S0034$\underline{71672007000500010}$

2. lida I. O que é ergonomia. In: IIDA, Itiro. Ergonomia: projeto e produção. $3^{\circ}$ ed. São Paulo: Edgard Blücher; 2005. P. 1-34

3. Carvalho GM. Enfermagem do Trabalho. Ed. Pedagógica e Universitária Ltda, Rio de Janeiro, $3^{a}$ reimpressão; 2001. P. $109 ; 113$

4. Carvalho R. Práticas Recomendadas SOBECC. Sobecc Assoc. Bras. de Enfermagem de Centro Cirúrgico Recuperação Anestésica e Centro de Material e Esterelização. São Paulo, $6^{a}$ edição; 2013. P.126-135

5. Tumm EMV, Macalai RT, Kirchner RM. Dificuldades enfrentadas por enfermeiros em um centro cirúrgico. Texto contexto - enferm. 2006;15(3):464-471. doi: 10.1590/ s0104-07072006000300011

6. Schmidt DRC, Dantas RAS. Qualidade de vida no trabalho de profissionais de enfermagem, atuantes em unidades do bloco cirúrgico, sob a ótica da satisfação. Rev. Latino-Am. Enfermagem. 2006;1 4(1):54-60. doi: 10.1590/S010411692006000100008

7. Silva VYNE, Karshiwabara TGB. Ergonomia aplicada à medicina no centro cirúrgico. Brazilian Journal of Surgery and Clinical Research - BJSCR, lpatinga- MG. 2013;4(3):41-44

8. Canzonieri AM. Metodologia da Pesquisa Qualitativa na Saúde. Petrópolis-RJ, $2^{a}$ edição, Editora Vozes; 2011 1. P. 37 38 e 47
9. Gerhardt TH, Silveira DT. (orgs). Métodos de Pesquisa. Porto Alegre: Editora da Universidade Federal do rio Grande do Sul; 2009

10. Minayo MCS. O Desafio do Conhecimento Pesquisa Qualitativa em Saúde. São Paulo, $14^{a}$ ed., Hucitec Editora; 2014. P. 303-309

11. Lopes MJM, Leal SMC. A feminização persistente na qualificação profissional da enfermagem brasileira. Cadernos pagu. 2005(24):105-125. doi: 10.1590/S0104$\underline{83332005000100006}$

12. Gomes LC, Dutra KE, Pereira ALS. O enfermeiro no gerenciamento do centro cirúrgico. Revista Eletrônica da Faculdade Metodista Granbery. 2014(16)

13. Magnag TSBS, Lisboa MTL, Griep RH, Kirchhof ALC, Guido LA. Aspectos psicossociais do trabalho e distúrbio musculoesquelético em trabalhadores de enfermagem. Rev. Latino-Am. Enfermagem 18(3). doi: 10.1590/S010411692010000300019

14. Junior $M H$, Goldenfum MA, Siena C. Lombagia ocupacional. Rev Assoc Med Bras 2010; 56(5): 583-9. doi: $10.1590 /$ S0104-42302010000500022

15. Vidor CR, Mahmud MAI, Farias LF, Silva CA, Ferrari JN, Comel JC et al. Prevalência de dor osteomuscular em profissionais de enfermagem de equipes de cirurgia em um hospital universitário. Acta Fisiatr. 2014;21 (1):6-10. doi: 10.5935/0104-7795.20140002

16. Leite PC, Silva A, Merighi MAB. A mulher trabalhadora de enfermagem e os distúrbios osteomusculares relacionados ao trabalho. Rev Esc Enferm USP. 2007;41(2):287-91. doi: $\underline{10.1590 / \text { S0080-62342007000200016 }}$

17. Silva BM, Lima FRF, Farias FSAB, Campos ACS. Jornada de trabalho: fator que interfere na qualidade da assistência de enfermagem. Texto Contexto Enferm. 2006;15(3):442-8. doi: $10.1590 /$ S0104-07072006000300008

18. Silva AP, Queiroz ES. O estresse e sua relação com a jornada de trabalho da enfermagem em unidade hospitalar. NBC - Periódico Científico do Núcleo de Biociências. 2011 ; 1 (1). doi: $10.15601 / 2238-1945 /$ penb.v ln1 p33-50

19. Pitta A. Hospital: dor e morte como ofício. 5.ed. São Paulo: Hucitec; 2003. P. 197

20. Azambuja EP, Pires DEP, Vaz MRC, Marziale MH. É possível produzir saúde no trabalho da enfermagem? Texto contexto - enferm. 2010;19(4). doi: 10.1590/S0104$\underline{07072010000400008}$

21. Elias MA, Navarro VL. A relação entre o trabalho, a saúde e as condições de vida: negatividade e positividade no trabalho das profissionais de enfermagem de um hospital escola. Rev. Latino-Am. Enfermagem. 2006;14(4) 
22. Linch GFC, Guido LA, Umann J. Estresse e Profissionais da Saúde: Produção do Conhecimento no Centro de Ensino e Pesquisas em Enfermagem. Cogitare Enferm. 2010;15(3).

23. Martino MMF, Misko MD. Estados emocionais de enfermeiros no desempenho profissional em unidades críticas. Rev. esc. enferm. USP. 2004;38(2).

24. Montanholi LL, Tavares DMS, Oliveira GR. Estresse: Fatores de risco não do Trabalho. Rev. bras. enferm. 2006;59(5). doi: 10.1590/S0034-71672006000500013

25. Pafaro RC, de Martino MMF. Estudo do estresse do enfermeiro com dupla jornada de trabalho em um hospital de oncologia pediátrica de Campinas. Rev. esc. enferm. USP. $2004 ; 38(2)$.

26. Oler FG et al. Qualidade de vida da equipe de enfermagem do centro cirúrgico. Arq Ciênc Saúde. 2005; $12(2): 102-10$.

27. Sulzbacher E, Fontana RT. Concepções da equipe de enfermagem sobre a exposição a riscos físicos e químicos no ambiente hospitalar. Rev. bras. enferm. 2013;66(1):25-30. doi: $10.1590 /$ S0034-71672013000100004

28. Almeida TRSH, Henrique MD, Moura MEL, Kirzner PL, Tavares KA, Pinto DS. Hérnia de Disco Lombar: Riscos e Prevenção. Rev. Ciênc. Saúde Nova Esperança. 2014;12(2). 